\title{
A patient with large congenital epulis in the maxillary and mandibular anterior areas accompanied by impaired suckling
}

\author{
Fumishige Oseko ${ }^{1}$, Toshiro Yamamoto ${ }^{1}$, Shigeta Takizawa ${ }^{1}$, Keiji Adachi ${ }^{1}$, and Narisato \\ Kanamura ${ }^{1}$ \\ ${ }^{1}$ Kyoto Prefectural University of Medicine
}

May 18, 2020

\begin{abstract}
Congenital epulis is a rare tumor of the the newborns. We found a case of soft elastic pedunculated masses in the left maxillary and mandibular anterior areas, protruding out of the oral cavity. They were diagnosed histopathologically and immunocamically as congenital granular cell epulis. These were no complication after surgery.
\end{abstract}

\begin{abstract}
Congenital epulis is a rare tumor of the the newborns. We found a case of soft elastic pedunculated masses in the maxillary (26 x $14 \times 11 \mathrm{~mm})$ and mandibular (55 x 44 x $11 \mathrm{~mm}$ ) anterior areas, protruding out of the oral cavity. The large multiple masses interfered with suckling. These masses were excised using a carbon dioxide laser under general anesthesia. They were diagnosed histopathologically and immunocamically as congenital granular cell epulis. These were no complication after surgery.
\end{abstract}

key words : congenital epulis,surgical excision,maxillary anterior,mandibular anterior

Key clnical message

Congenital epulis, which are soft elastic pedunculated masses in the left maxillary and mandibular anterior areas protruding out of the oral cavity, is a rare tumor of the the newborns. These masses were no complication after dioxide laser surgery.

1. Introduction

Congenital epulis is a localized benign mass that develops in the gingiva of newborns and it was initially reported by Neumann in 1871. To our knowledge, approximately 200 cases have been reported, but development in the upper and lower jaws is very rare.

We report a patient with large congenital epulis, which developed in the gingiva of the left maxillary and mandibular anterior tooth regions.

2. Case report

Patient: Newborn at one day after birth

First examination: May 2013

Chief complaint: Impaired suckling.

Familial medical history: None in particular 
History of present illness: No abnormalities were noted during pregnancy. The patient was delivered normally (3,094 g, 38 weeks and 5 days of gestation) and the Apgar score was 10/10. As masses were noted in the left maxillary and mandibular anterior tooth regions at birth, the patient was transferred from an obstetric clinic to a nearby emergency hospital. No concomitant physical or visceral malformation was observed, but the masses were judged as requiring treatment. The patient was transferred to the NICU of our hospital and then referred to our department for close examination of the masses.

Present status:

Systemic findings: None in particular

Local findings: In the oral cavity, elastic soft pedunculated masses, with sizes of $26 \times 14 \mathrm{x} 11$ and $55 \mathrm{x} 44 \mathrm{x}$ $11 \mathrm{~mm}$, were present in the left maxillary and mandibular anterior tooth regions, respectively. The masses extraorally protruded. The surfaces of the maxillary and mandibular masses were smooth, their color was bright red, and flare was partially noted (Fig. 1).

Imaging findings: On MRI, both maxillary and mandibular masses demonstrated slightly higher intensity than muscle on T1-weighted imaging, and $20 \times 15 \times 10-\mathrm{mm}$ and $50 \times 40 \times 10-\mathrm{mm}$ solid masses exhibiting slightly high intensity were detected on T2-weighted imaging. Their content was mostly homogeneous.

Clinical diagnosis: Congenital epulis

Treatment and course: After admission to the NICU, tube feeding was performed for impaired suckling under whole body management by the pediatric department and the respiratory condition was stable. As necrosis of the mass surface progressed, the epulis was resected by carbon dioxide laser for the clinical diagnosis of congenital epulis under general anesthesia 5 days after admission. The resected specimen was solid elastic and soft. After surgery, no problems, such as infection, occurred. Training of suckling was initiated 3 days after surgery and the patient was discharged at 10 days with a favorable recovery (Fig. 2).

Histopathological findings: Under the mucosal epithelium, solid cells containing cytoplasm filled with eosinophilic granules and oval-shaped dark-stained nuclei with a clear nucleolus had densely proliferated. The interstitium comprised narrow vascular connective tissue, and hemorrhage and necrosis were partially noted. The superficial layer was covered with squamous epithelium, demonstrating parakeratosis and prickle cell hypertrophy.

On immunohistochemical staining, the masses were PAS staining $(+)$, alcian blue staining $(+)$, NSE $(+)$, S-100 protein (-), Vimentin (+), CD68 (-), neurofilament (-), and GFAP (-).

Histopathological diagnosis: Congenital granular cell epulis.

\section{Discussion}

Congenital epulis is a rare disease developing in newborns. It was initially reported by Neumann in 1871[1] and approximately 200 cases have been reported[2]. The incidence is higher in females at a sex ratio of female: male $=8: 1$. It is more frequently observed in maxillary than mandibular gingival mucosa $(2: 1$ or $3: 1)$, and lesions are bright red, elastic soft, and pedunculated with a smooth surface[3,4]. The size of lesions varies up to $90 \mathrm{~mm}[3]$.

Course observation may be prioritized when there is no functional or esthetic disturbance. In the present patient, the lesions were large congenital epulis that developed on the same side of the upper and lower jaws, and as impaired suckling and esthetic disturbance were observed, surgical resection was indicated. No case of recurrence after resection has been reported, but extensive resection may cause eruption disorder of deciduous teeth[5], to which attention should be paid.

The main diseases included in the differential diagnosis in newborns are Epstein pearl, granular cell tumor, vascular malformation, and neuroectodermal tumor in childhood. The histopathological findings of congenital epulis are particulate granules, eosinophilic cytoplasm, and slightly abnormal nuclei. No pseudocarcinomatous hyperplasia is observed in the capsular mucosa in congenital epulis in contrast to granular cell 
tumors[6]. Similar findings were noted in the present patient. Immunohistologically, congenital epulis and granular cell tumors are S100 protein $(-)$ and vimentin $(+)[7]$, although vimentin (-) granular cell tumors have been reported[10]. In addition, granular cell tumors are NSE (+), GFAP (-), CD68 (-), and desmin $(-)[7]$. Both types of lesions are PAS staining $(+)$ in many cases[6].

In the present patient, cytoplasm with eosinophilic granules was noted on HE staining, and the cells contained glycoprotein based on PAS staining $(+)$ and Alcian blue staining $(+)$. On immunohistochemistry, the masses were found to have originated from granular cells because they were NSE $(+)$, S-100 protein (-), vimentin $(+), \operatorname{GFAP}(-), \operatorname{CD}-68(-)$, and desmin (-).

Many points remain unclear regarding the pathogenesis of this disease. Fibroblasts, histiocytes, myogenic, neurogenic, reactive proliferation, and hamartoma of mesenchymal cells have been reported as the tissue of origin [2,3]. Miura et al. [8] considered mechanical stimulation of the oral mucosa by the finger tips and nails, such as sucking, to be behaviors exhibited in the fetal period that can induce epulis. Furthermore, Mori et al. [9] reported that fetuses in the uterus start sucking behavior at approximately 17 weeks of gestation, and the sucking force is sufficiently strong to form sucking blisters on the 1st and 3rd fingers, wrist, and dorsum of the hand. Thus, epulis formation may be consistent with inflammatory reactions caused by sucking stimulation.

In a study by Ohkawa et al. [10], congenital epulis was accompanied by granular cells in all cases treated by resection earlier than 20 days after birth, but it was not accompanied by granular cells in cases at 2 or more months after birth, and both types were present in cases treated surgically between 20 days and 2 months after birth. They considered the internal environment, such as hormonal and immunological factors, to change after birth, causing granular cells to disappear and be replaced with fibrous connective tissue. This disappearance of granular cells and replacement with fibrous connective tissue may occur after birth, i.e., transition to fibrous connective tissue does not occur in the fetal environment.

In cases of spontaneous regression after birth, the final step of inflammation, absorption and replacement (tissue regeneration) of inflammatory tissue, may have occurred after birth. Based on the above findings, mechanical stimulation starts from 17 weeks of gestation, at which sucking starts and the epulis is formed through inflammation. Moreover, the final step of inflammation, absorption and replacement, does not progress during the fetal period. Therefore, the epulis grows to a large size because mechanical stimulation by sucking starts earlier.

In our patient, surgical resection was selected because impaired suckling and esthetic disturbance were noted due to large lesions in the upper and lower jaws on the same side, and a favorable clinical course was acquired. As the delayed eruption of deciduous teeth and malalignment may occur in the future, long-term follow-up is important.

\section{Conclusion}

We report a 1-day-old newborn girl with large congenital epulis in the left anterior deciduous tooth regions of the upper and lower jaws, accompanying impaired suckling. The histopathological finding of the masses was congenital granular cell epulis.

There is no conflict of interest for this report.

Fig. 1

Intraoral Figgraph 5 days after admission: Pedunculated tumorous lesions with sizes of $26 \times 14 \times 11$ and 55 x $44 \times 11 \mathrm{~mm}$ were present in the left maxillary and mandibular anterior tooth regions, respectively. The mass surface was necrotized.

Fig. 2

Intraoral Figgraphs 10 days after surgery

References 
1) Neumann E: Ein von congenital epulis of newborn. Arch Heilkd.1871; 12: 189-190.

2) Nakata M, Annol K, et al: Prenatal diagnosis of congenital epulis: A case report. Ultrasound Obstet Gynecol. 2002; 20: 627-629.

3) Chami RG, Wang HS: Large congenital epulis of newborn. J Pediatr Surg. 1986; 21: 189-190.

4) Bowe JJ: Congenital epulis tumor. Case report. Plast Reconstr Surg. 1974; 5: 227-229.

5) Küpers AM, Andriessen P, et al: Congenital epulis of the jaw: a series of five cases and review of literature. Pediatr Surg Int.2009; 25: 207-210.

6) Prigkos AC, Nikolakis MD, et al:Spindle cell epulis in an 8-month-old child: a histologic variant of congenital granular cell epulis? Head Neck Pathol. 2012; 6: 467-470.

7) Sahu S, Maurya R, et al:Multiple congenital epulis in newborn - A rare presentation. J Oral Maxillofac Pathol . 2009; 13: 78-80.

8) Miura M,Takagishi M, et al :A case of congenital epulis in a neonate.POMS. 2006; 16: 36-39.

9) Mori G :Natural history of sucking blisters. Obstetrics and Gynecology. 1990; 6: 1361-1365.

10) Ohkawa M, Hiramoto M, et al : A case of congenital epulis which decreased in size. Practica Oto-RhinoLaryngologica. 1981; 75: 329-355.

\section{AUTHOR CONTRIBUTIONS}

Fumishige Oseko and Toshiro Yamamoto: have made substantial contribution to conception and design of the manuscript as they interpreted data, drafted the work, approved the final version, and agreed to be accountable for all aspects of the work. Shigeta Takizawa and Keiji Adachi: have been involved in drafting the manuscript, approving the final ver-sion, and agreed to be accountable for all aspects of the work. Narisato Kanamura: has critically revised the work, given the final approval of the version to be published, and agreed to be accountable for all aspects of the work.

\section{Hosted file}

Fig1.pptx available at https://authorea.com/users/322962/articles/451933-a-patient-withlarge-congenital-epulis-in-the-maxillary-and-mandibular-anterior-areas-accompanied-byimpaired-suckling

\section{Hosted file}

Fig 2.pptx available at https://authorea.com/users/322962/articles/451933-a-patient-withlarge-congenital-epulis-in-the-maxillary-and-mandibular-anterior-areas-accompanied-byimpaired-suckling 